\title{
Orientação ao usuário de prótese auditiva: retenção da informação
}

\section{Guidance to the user of the hearing aid: retention of information}

\author{
Tatiane Geraldo", Deborah Viviane Ferrari², Barbara Guimarães Bastos ${ }^{3}$. \\ 1) Especialista em Audiologia Clinica e Educacional - HRAC/USP. Fonoaudióloga. Politec Saúde. \\ 2) Doutorado. Professor Doutor. \\ 3) Fonoaudióloga. Discente do Programa de Mestrado em Fonoaudiologia da Faculdade de Odontologia de Bauru - Universidade de São Paulo. \\ Instituição: Centro de Pesquisas Audiológicas. Hospital de Reabilitação das Anomalias Craniofaciais - Universidade de São Paulo. Campus Bauru. \\ Bauru / SP - Brasil. \\ Endereço para correspondência: Departamento de Fonoaudiologia. - Al. Octávio Pinheiro Brizolla 9-75 - Vila Universitária - Bauru / SP - Brasil - CEP: 17012-901 - Telefone: \\ (+55 14) 3235-8332 - E-mail: deborahferrari@usp.br \\ Artigo recebido em 14 de Fevereiro de 2011. Artigo aprovado em 18 de Agosto de 2011
}

\section{RESUMO}

Introdução: Diferentes informações são transmitidas ao novo usuário de prótese auditiva, em um curto período de tempo. O usuário necessita entender e reter tais informações para subsequente recuperação e ação, assegurando o uso apropriado do dispositivo.

Objetivo: Verificar a retenção de informações sobre a perda auditiva e uso/cuidados com a prótese auditiva em novos usuários destes dispositivos. Analisar se existe influência de dados demográficos e audiológicos neste processo.

Método: Estudo prospectivo. Participantes: 30 deficientes auditivos (18 mulheres e 12 homens) com idades entre $18 \mathrm{e}$ 88 anos. Foram oferecidas orientações verbais apoiadas com uso de ilustrações no momento do diagnóstico audiológico e adaptação da prótese auditiva. Houve treino de manipulação da prótese. Três semanas após a adaptação foi realizada a avaliação da retenção da informação sobre a perda auditiva (evocação livre) e uso/cuidados com a prótese (evocação auxiliada). A manipulação das próteses também foi avaliada. As respostas e observações do avaliador foram anotadas em um protocolo específico e pontuadas.

Resultados: Em média os participantes retiveram, respectivamente, $31,6 \%$ e $83,6 \%$ das informações sobre a perda auditiva e uso/cuidados com a prótese auditiva. Houve diferença significativa da retenção de informação sobre a perda auditiva entre adultos e idosos. Não houve correlação entre a retenção de informação com o grau da perda auditiva, escolaridade e nível sócio econômico.

Conclusão: Faz-se necessária à utilização de estratégias que facilitem e melhorem a retenção da informação sobre o diagnóstico audiológico, uso e cuidados com a prótese auditiva para novos usuários deste dispositivo.

Palavras-chave: audição, perda auditiva, aconselhamento, auxiliares de audição.

\section{SUMMARY}

Introduction: Different informations are transmitted to the new user of the hearing aid, in a short period of time. The user needs to understand and retain the information to subsequent recovery and action, ensuring the proper use of the device. Objective: check the information retention about the hearing loss and use/care with the hearing aid in new users of these devices. Examine whether there is influence of demographic data and audiologic.

Method: Prospective study. Participants: 30 hearing impaired (18 women and 12 men), aged between 18 and 88 years. Were offered verbal orientation supported with ilustrations at the time of audiological diagnosis and adaptation of the hearing aid. There was practice for manipulation of the prosthesis. Three weeks after the adaptation, was performed a evaluation of the retention of information about hearing loss (free recall) and use/care with the prosthesis (aided recall). The manipulation of the prosthesis was also evaluated. The responses and comments of the evaluator were noted in a specific protocol and scored.

Results: On average the participants retained, respectively, $31,6 \%$ and $83,6 \%$ of informations about hearing loss in adults and elderly. There was no correlation between the retention of information with the degree in hearing loss, education and socioeconomic level.

Conclusion: It's necessary the use of strategies that facilitate and improve the retention of the information about the audiological diagnosis, use and care with the hearing aid for new users of this device.

Keywords: hearing, hearing loss, counseling, hearing aids. 


\section{INTRODUÇÃO}

Fornecer aconselhamento a clientes e seus familiares e/ou cuidadores é uma das áreas de competência do fonoaudiólogo. Oaconselhamento é uma oportunidade de receber e fornecer informações de modo a facilitar o entendimento da deficiência auditiva e o ajuste a esta condição. De modo geral dois tipos de aconselhamento podem ser oferecidos, sendo ambos importantes no processo de adaptação da prótese auditiva: (1) ajuste pessoal, o qual envolve o desenvolvimento de mecanismos e sistemas de suporte emocional para o indivíduo lidar com a deficiência e (2) informativo, aqui considerado como sinônimo de "orientação" (1).

No que diz respeito à orientação sugere-se a abordagem dos seguintes tópicos: anátomo-fisiologia da audição; natureza dos diferentes tipos de deficiência auditiva; características gerais das próteses auditivas e explicação dos motivos que nortearam a seleção de um tipo de prótese em particular; instruções sobre o uso, cuidado e resolução de problemas com as próteses auditivas e molde auricular; instruções sobre o uso do telefone e equipamento auxiliares, quando pertinente; recomendações para as consultas de acompanhamento dos adultos e orientação a respeito das expectativas quanto ao uso da prótese auditiva $(2,3)$. Também é enfatizada a importância de demonstrações manuais de como operar a prótese auditiva (4).

Estas informações são importantes para assegurar o uso apropriado das próteses auditivas e manter o bom funcionamento do dispositivo, evitando a necessidade de reparos ou reposições e, sobretudo, garantindo que o sinal amplificado seja o mais claro possível para o usuários (5).

Como visto, existe uma variedade de informações que devem ser transmitidas e demonstradas ao usuário da prótese auditiva, geralmente em um curto período de tempo. Este indivíduo, por sua vez, necessita entender e armazenar acuradamente tais informações, muitas vezes não familiares, para subsequente recuperação e ação (6).

Dentre outros fatores, a ansiedade causada pela situação de utilizar uma prótese auditiva, a falta de familiaridade com o conteúdo abordado na orientação e a própria presença de uma perda auditiva dificultam o entendimento da mensagem e retenção da informação. Além disto, as habilidades comunicativas do profissional, as estratégias utilizadas para transmitir a informação bem como a quantidade de informações oferecidas influenciam o processo de retenção das orientações (2, 7-9).

Outro ponto importante é o tipo de tarefa utilizada para avaliar a habilidade do paciente em reter informações de saúde. Nas tarefas de reconhecimento o indivíduo necessita selecionar a informação correta dentre diferentes opções fornecidas-similar a um teste de múltipla escolha. Em tarefas de evocação auxiliada (probed recall) existe algum tipo de pista ou auxílio para evocação. Por exemplo: "Qual é o tamanho da bateria da sua prótese auditiva?". Em tarefas de evocação livre nenhuma pista é fornecida, por exemplo, o indivíduo necessitaria evocar o tamanho da bateria da prótese frente à pergunta "Me diga tudo o que você sabe sobre o uso e cuidado da sua prótese auditiva" (10).

Poucas pesquisas identificaram a quantidade de informação retida após as orientações na clínica audiológica. Em um estudo com adultos novos usuários, empregando tarefas de reconhecimento, foi verificado que estes que retinham aproximadamente 85 a 75\% da informação fornecida imediatamente ou após os primeiros meses de adaptação da prótese auditiva. Foi observado, ainda, que a idade e severidade da perda auditiva diminuíam a retenção da informação (10). Em outro estudo, adultos novos usuários responderam um questionário com 25 perguntas abertas (tarefa de evocação auxiliada) sendo observado que em média a retenção da informação foi de 80\% e 77\% imediatamente e quatro semanas após a adaptação, respectivamente (11).

A observação de como o usuário cuida e manipula a prótese auditiva e molde auricular fornece uma medida indireta de como a orientação fornecida foi retida e está sendo utilizada. Oliveira et al. (2001) (12) verificaram que $76 \%$ de adultos novos usuários apresentaram alguma dificuldade de manipulação e uso da prótese e/ou molde auricular um mês após a adaptação. Em outro estudo foi observado que a maior parte dos usuários tinha dificuldades em relação aos moldes auriculares ou cápsulas da prótese auditiva (13). No caso de usuários experientes também foi demonstrado que o nível de habilidade dos mesmos quanto à manipulação e cuidados com a prótese auditiva variou de excelente a ruim, sendo que piores desempenhos foram observados em tarefas de limpeza da prótese e uso da bobina telefônica (5).

Oentendimentoe a retençãoda informaçãofornecida pelo profissional de saúde aumentam a satisfação do paciente e a aderência ao tratamento ao mesmo tempo em que diminui a ansiedade e o tempo de tratamento o que, por consequência, diminui também o seu custo (3). Estudos realizados com idosos demonstraram que a facilidade de manipulação e cuidados com a prótese auditiva e molde auricular estava relacionada com o número de horas de uso e a autopercepção do benefício obtido com o uso do dispositivo $(14,15)$.

Pelo exposto, o objetivo deste estudo é verificar o quanto adultos novos usuários de prótese auditiva conse- 
guem reter das orientações oferecidas após o diagnóstico audiológico e processo de adaptação da prótese auditiva e analisar se fatores como a idade, o grau da perda auditiva, o nível sócio econômico e a escolaridade apresentam alguma influência na retenção da informação.

\section{MÉTODO}

O presente estudo prospectivo, descritivo, exploratório e transversal foi realizado no Centro de Pesquisas Audiológicas (CPA) do Hospital de Reabilitação de Anomalias Craniofaciais (HRAC) da Universidade de São Paulo, sendo a provado pelo Comitê de Ética em Pesquisas com Seres Humanos (protocolo 099/2005-CPq). Todos os participantes foram voluntários, concordaram com a realização dos procedimentos necessários para a execução do estudo e assinaram o Termo de Consentimento Livre Esclarecido.

\section{Participantes}

O estudo envolveu uma amostra selecionada (não aleatória) correspondente aos primeiros 30 indivíduos atendidos no serviço em questão que aceitassem participar voluntariamente da pesquisa e obedecessem aos seguintes critérios de inclusão: (a) apresentar idade maior que 18 anos, (b) ser portador de deficiência auditiva de qualquer tipo ou grau, uni ou bilateral, (c) não apresentar deficiência visual que não fosse passível de correção com lentes, (d) não apresentar alterações motoras ou alterações de destreza manual graves, (e) não apresentar processos demenciais ou outros distúrbios neurológicos ou psiquiátricos (conforme avaliação neurológica e psicológica constantes no prontuário dos participantes), (f) não ter realizado exames audiológicos anteriormente, quer em serviço público ou privado e (g) não apresentar experiência prévia com uso da prótese auditiva.

Os participantes possuíam idade variando entre 18 e 88 anos, sendo 13 adultos (idade menor que 60 anos) e 17 idosos. Dezoito participantes eram do sexo feminino e 12 do sexo masculino. Nenhum participante havia passado pelo processo de diagnóstico audiológico anteriormente, quer seja em serviços públicos ou privados. Todos apresentavam deficiência auditiva pós-lingual sendo neurossensorial bilateral $(n=26)$, mista bilateral $(n=3)$ e neurossensorial em uma orelha e mista na outra $(n=2)$. Vinte participantes possuíam perda auditiva simétrica e 10 possuíam perda assimétrica. A classificação do grau da perda auditiva foi realizada a partir da média dos limiares de $500,1 \mathrm{k}, 2 \mathrm{k}$ e $4 \mathrm{k} \mathrm{Hz}$ na melhor orelha, de acordo com a Organização Mundial de Saúde (16). Todos os participantes eram novos usuários e foram adaptados com próteses auditivas digitais ( $n=27,90 \%)$, analógicas $(n=1,3,3 \%)$ e híbridas ( $n=2,6,7 \%)$. Os dados de escolaridade e classificação sócio-econômica (17) foram extraídos do formulário de avaliação do serviço social que constava no prontuário dos participantes. A caracterização dos participantes encontrase na Tabela 1.

\section{Procedimentos}

O processo de orientação foi realizado em diferentes etapas, sendo o conteúdo abrangido em cada uma delas descrito Tabela 2. O participante compareceu ao Centro de Pesquisas Audiológicas em três dias consecutivos. No primeiro dia foi realizado o diagnóstico, no segundo dia foram feitos os procedimentos de seleção e verificação da prótese e no terceiro dia foi realizada a adaptação.

Para a realização das atividades de orientação foi feita a transformação do discurso técnico em discurso comum, sendo utilizados apoios visuais como, por exemplo, figuras e gráficos específicos para facilitar a compreensão da mensagem. Estratégias de comunicação compatíveis com as habilidades auditivas do participante foram também empregadas (por exemplo: uso de leitura orofacial, diminuição da velocidade da fala, uso de repetições, refraseamento, apoio da escrita quando necessário).

Um mesmo profissional realizou as orientações para todos os participantes. Uma lista de verificação foi utilizada para que este profissional abordasse todos os tópicos necessários. Os participantes foram solicitados a interromper o avaliador e solicitar clarificação sempre que necessário.

Tabela I. Caracterização dos participantes $(n=30)$.

\begin{tabular}{|c|c|c|c|c|c|c|c|c|c|c|c|c|c|}
\hline \multicolumn{3}{|c|}{ Idade } & \multicolumn{3}{|c|}{ Grau da Perda } & \multicolumn{2}{|c|}{ Tipo de Prótese Auditiva } & \multicolumn{3}{|c|}{ Classificação Sócio Econômica } & \multicolumn{3}{|c|}{ Escolaridade } \\
\hline$x$ & $d p$ & Leve & Mod & Sev & Prof & Retro & Intra & BS & $\mathrm{Ml}$ & $\mathrm{Me}$ & $\mathrm{Fl}$ & $M$ & $\mathrm{~S}$ \\
\hline 52 & 14 & 8 & 18 & 3 & I & 12 & 18 & 18 & $\mid 1$ & I & 15 & 10 & 5 \\
\hline
\end{tabular}

Legenda: Mod: Moderada Sev: Severa Prof: Profunda

Retro: Retroauricular Intra: Intra-canal BS: Baixa superior MI: Média Inferior Me: Média FI: Fundamental Incompleto M: Médio Completo S: Superior completo. 
Tabela 2. Informações oferecidas aos participantes do estudo.

\begin{tabular}{|c|c|}
\hline Etapa & Conteúdo \\
\hline I. Após diagnóstico audiológico & $\begin{array}{l}\text { a) o processo de audição normal; } \\
\text { b) tipo e grau de deficiência auditiva que o indivíduo possui; } \\
\text { c) provável etiologia da deficiência auditiva, quando esta é determinada; } \\
\text { d) opções de tratamento (médico, cirúrgico ou clínico-reabilitativo)* }\end{array}$ \\
\hline \multicolumn{2}{|c|}{ 2. Após o processo de seleção e verificação do } \\
\hline benefício da prótese auditiva. & $\begin{array}{l}\text { a) Resultados obtidos nas diferentes avaliações: audiometria em campo livre, } \\
\text { medidas com microfone sonda e testes de percepção da fala; } \\
\text { b) Expectativas com relação ao uso da prótese auditiva. }\end{array}$ \\
\hline 3. Adaptação da prótese auditiva. & $\begin{array}{l}\text { a) Inserção e remoção da pilha; função do lacre da pilha; inserção e remoção } \\
\text { da prótese auditiva e molde auricular; higienização; manipulação do controle } \\
\text { de volume; uso de estratégias de comunicação. }\end{array}$ \\
\hline
\end{tabular}

*As opções de tratamento foram discutidas juntamente com um médico otorrinolaringologista.

O tempo utilizado para a orientação sobre as características da deficiência auditiva e sobre uso e cuidados com a prótese auditiva foi semelhante, em torno de 40 minutos. Durante a sessão de orientação cada um dos participantes praticou juntamente com o fonoaudiólogo as atividades de inserção e remoção do molde auricular / prótese auditiva na orelha, inserção e remoção da bateria, operação do controle de volume e chaves comutadoras (quando existentes) e uso do telefone. As orientações de manipulação, uso, limpeza e cuidados com a prótese auditiva foram também oferecidas na forma escrita (manuais do próprio fabricante), possibilitando ao participante a leitura das mesmas, em sua residência, após a adaptação.

Os participantes retornaram ao serviço para a primeira consulta de acompanhamento, quando foi realizada a avaliação da retenção da informação fornecida. O tempo discorrido entre a adaptação da prótese e a avaliação foi igual a uma $(n=3)$, três $(n=21)$ e quatro semanas $(n=6)$. A diferença entre os tempos para a avaliação ocorreu em função da disponibilidade de comparecimento do participante.

Para o propósito desta avaliação foi elaborado um protocolo contendo informações gerais do modelo, tipo e tecnologia empregada na prótese auditiva adaptada, número de horas diárias de uso do dispositivo e os itens abordados nas diferentes etapas de orientação. O avaliador realizava uma entrevista com cada participante, questionando-o a respeito das características da sua perda auditiva bem como quanto ao uso e cuidados com a prótese auditiva. O avaliador também realizava a inspeção das condições de uso e conservação do molde auricular e da prótese auditiva.

No que se refere às características da perda auditiva o paciente foi solicitado a relatar qualquer informação que recordasse (tarefa de evocação livre). As informações de interesse a serem pontuadas se referiam à severidade da perda auditiva (grau da perda) e localização da lesão (tipo da perda). Não era esperado dos mesmos o uso de terminologia técnica. As respostas do participante foram registradas no protocolo. Dois avaliadores independentes analisaram tais respostas e atribuíram uma nota. Se o participante recordasse corretamente as informações era atribuída a pontuação 10, se recordasse parcialmente (por exemplo, se recorda da severidade da mesma mas não do local da lesão) era atribuída a pontuação 5 e no caso de não se recordar de nenhuma informação era atribuída à pontuação 0 .

Para a avaliação da retenção da informação quanto ao uso e cuidado com a prótese foi utilizada tarefa de evocação auxiliada. Foram realizadas perguntas a respeito de dez itens: inserção e remoção da bateria, duração da bateria, necessidade de manter uma bateria extra para troca, remoção da prótese auditiva/molde, inserção da prótese auditiva/molde, higienização do molde e prótese, manipulação do controle liga/desliga, cuidados com a prótese auditiva e molde auricular e resolução de problemas com a prótese auditiva. O participante também era solicitado a manipular a prótese auditiva em frente ao avaliador (por exemplo, colocar e retirar molde auriculare/ ou prótese auditiva, colocar e retirar as pilhas, manipular controle de volume e chaves comutadoras de liga e desliga, bobina telefônica, etc). Desta forma, eram consideradas tanto a resposta verbal do participante quanto à demonstração da ação. Foi atribuído um ponto para cada item respondido ou demonstrado corretamente.

As pontuações obtidas por cada participante foi dividida pela pontuação total possível (igual a 10) e multiplicado por 100 , a fim de obter a porcentagem de informações retidas.

Com relação à manipulação do controle de volume este item foi analisado separadamente em virtude de existir 18 participantes que utilizavam prótese auditiva intracanal com controle de volume automático. 
A análise dos dados foi realizada por meio de estatística descritiva. Foram também realizadas comparações das médias das pontuações de reconhecimento da informação entre homens e mulheres, entre adultos e idosos e entre usuários de prótese retroauricular e intracanal (teste t). Foram verificadas as correlações entre o grau da perda auditiva (coeficiente de correlação de Pearson), escolaridade e nível sócio econômico dos participantes (coeficiente de correlação se Spearman) e as pontuações obtidas pelos indivíduos durante a avaliação. Para todos os casos foi adotado alfa igual a $5 \%$.

\section{RESULTADOS}

O Gráfico 1 mostra os resultados obtidos com relação à retenção da informação sobre as características da deficiência auditiva.

Com relação ao número de horas de uso da prótese auditiva, 22 participantes $(73,3 \%)$ a utilizavam por mais que dez horas e oito participantes (26,7\%) a utilizavam por até quatro horas diárias. Destes últimos, um indivíduo não utilizava a prótese durante sua jornada de trabalho, uma vez o ambiente apresentava muita umidade e poeira, e três indivíduos ainda estavam no período de aclimatização aos sons amplificados.

O Gráfico 2 mostra a distribuição da pontuação obtida para os itens de uso, cuidados e manipulação da prótese auditiva e/ou molde auricular.

Com relação à manipulação do controle de volume realizada pelos 12 usuários de prótese auditiva retroauricular, verificou-se que nove participantes (75\%) realizavam manipulação adequada e três (25\%) não se recordavam da função deste controle e, portanto, não o manipulavam embora sentissem a necessidade de aumentar ou diminuir a amplificação em alguns ambientes.

A diferença entre as médias da pontuação de uso e cuidados com a prótese entre usuários de dispositivos retroauriculares $(\mathrm{x}=8,3 ; \mathrm{dp}=2)$ e intracanais $(\mathrm{x}=9,1 ; \mathrm{dp}=1,2)$ não foi estatisticamente significativa $(\mathrm{p}=0,12)$.

As médias da pontuação entre adultos e idosos bem como a significância estatística entre elas (teste t) encontrase na Tabela 3 .

A Tabela 4 mostra as correlações obtidas entre a escolaridade, classificação sócio-econômica e grau da perda auditiva dos participantes com a retenção da informação a respeito das características da perda auditiva e uso e cuidados com a prótese auditiva e molde auricular.

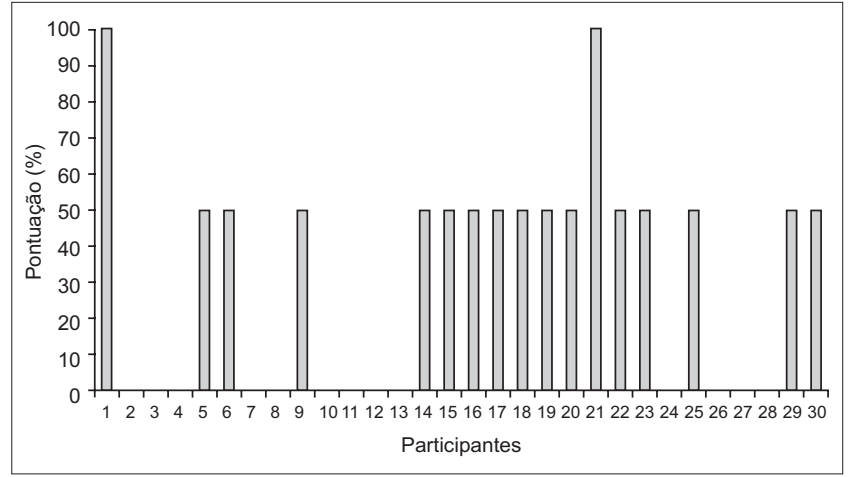

Gráfico 1. Distribuição das pontuações obtidas quanto à retenção da orientação a respeito das características da perda auditiva $(n=30)$.

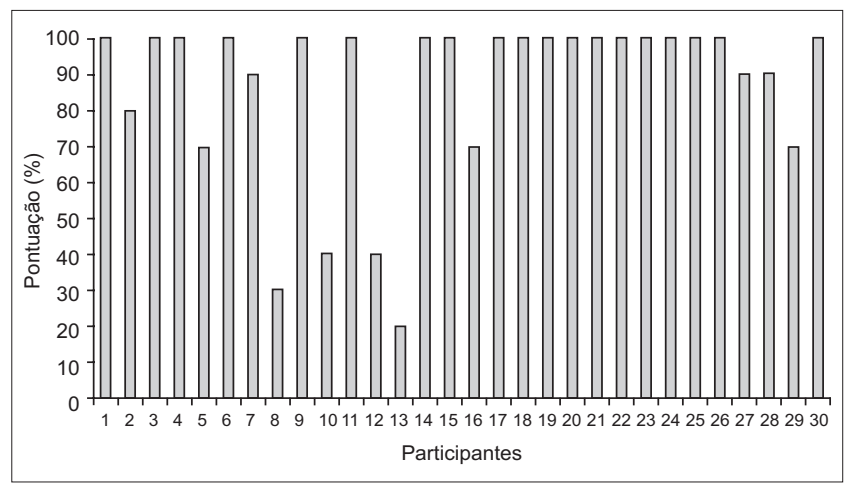

Gráfico 2. Distribuição das pontuações obtidas quanto ao uso, cuidados e manipulação da prótese auditiva/molde auricular $(n=30)$.

Tabela 3. Médiae desvio padrão da quantidade de informação retida quanto à faixa etária e o valor da significância estatística $(n=30)$.

\begin{tabular}{|c|c|c|c|c|c|}
\hline & \multicolumn{5}{|c|}{ Faixa etária } \\
\hline & $x$ & $\mathrm{dp}$ & $x$ & $\mathrm{dp}$ & $\mathrm{p}$ \\
\hline Perdaauditiva & 5 & 2,8 & I,7 & 2,4 & $0,001 *$ \\
\hline Prótese auditiva & 8,7 & 1,9 & 8,8 & 1,4 & 0,13 \\
\hline
\end{tabular}

* $\mathrm{p}<0,005$ estatisticamente significante.

Tabela 4. Correlações entre a escolaridade, classificação sócio-econômica(Spearman)egrau daperda auditiva(Pearson) dos participantes com a retenção da informação a respeito das características da perda auditiva e uso e cuidados com a prótese auditiva $(\mathrm{n}=30)$.

\begin{tabular}{lcccccc}
\hline & Escolaridade & \multicolumn{2}{c}{$\begin{array}{c}\text { Classificação } \\
\text { Sócio- } \\
\text { Econômica }\end{array}$} & $\begin{array}{c}\text { Grauda } \\
\text { perda } \\
\text { auditiva }\end{array}$ \\
\hline rho & $P$ & rho & $P$ & $r$ & $p$ \\
\hline Perdaauditiva & 0,16 & 0,37 & 0,09 & 0,62 & $-0,05$ & 0,77 \\
Prótese auditiva & 0,18 & 0,34 & 0,13 & 0,48 & $-0,17$ & 0,35
\end{tabular}

${ }^{*} \mathrm{p}<0,005$ estatisticamente significante. 


\section{DISCUSSÃO}

Em média, os participantes conseguiram reter $31,6 \%(\mathrm{dp}=30)$ das informações a respeito das características de sua perda auditiva (Gráfico 1 ). Verificou-se que apenas dois indivíduos $(6,7 \%)$ conseguiram evocar 100\% das informações fornecidas, incluindo a hipótese etiológica. Doze participantes (40\%) conseguiram evocar apenas a severidade da perda. A análise das respostas registradas mostrou ser comum a referência a uma porcentagem de perda da audição, embora esta informação não tivesse sido fornecida na sessão de orientação: "(.) perdi 60\% dos agudos"; "é uma perda de 50\% nas duas orelhas", "(...) é leve, perdi uns 10\% da audição (...)".

Três participantes (10\%) conseguiram evocar apenas o local da lesão "(..) é nas células da cóclea"; "o problema está no ouvido mais interno", "(..) perfuração no tímpano e também na cóclea".

De particular importância foi o fato de que 13 participantes (43,3\%) não conseguiram evocar corretamente nenhuma informação a respeito das características de sua deficiência auditiva.

Até a conclusão deste artigo não havia sido encontradas outras pesquisas a respeito da retenção da informação sobre a perda de audição. A análise da literatura em outras áreas da saúde mostra que é comum os pacientes não reterem informações a respeito do diagnóstico de uma doença, até mesmo quando esta ameaça a vida. Na área de oncologia verificou-se que dez dias após o diagnóstico os pacientes conseguiam evocar 50\% das informações fornecidas (18). Além disto, uma pesquisa na área de emergência médica observou que cerca de 80\% dos pacientes compreendia erroneamente as informações diagnósticas fornecidas sem, no entanto, estarem cientes disto (19).

Com relação às informações de uso e cuidados com a prótese auditiva, em média os participantes evocaram 86,3\% das informações (Gráfico 2). Ressalta-se que a inserção e remoção da bateria e lacre foi o item em que os participantes tiveram mais dificuldade, sendo que apenas 14 participantes (47\%) conseguiram evocar esta informação corretamente.

Devido às diferenças metodológicas não é possível realizar uma comparação direta do presente estudo com a literatura, no entanto, os dados aqui relatados estão em concordância com outros estudos com novos usuários de AASI onde foram observadas retenção de informação de 77 a $85 \%(10,11)$.
Os participantes evocaram maior número de informações sobre o uso e cuidados com a prótese auditiva do que sobre as características de sua deficiência auditiva. Um dos fatores que contribuiu para este resultado foi o tipo de tarefa utilizada na avaliação. O nível de dificuldade associada com uma dada tarefa é atribuído à quantidade de esforço necessário para a recuperação da informação. Assim, tarefas de livre evocação são mais difíceis do que tarefas de evocação auxiliada (11).

Outro fator que pode ter contribuído é o impacto do diagnóstico da deficiência auditiva que, frequentemente, gera no indivíduo sentimentos de choque, dor e estresse (20). Estes sentimentos contribuem para o estreitamento da atenção(8) dificultando a absorção de informação e fazendo com que o indivíduo se recorde apenas do dado mais importante e de maior impacto emocional(7). Ou seja, quando confrontado com o diagnóstico da deficiência auditiva, geralmente incurável e com consequências para toda vida, esta mensagem pode ter se tornado o foco de atenção do indivíduo e as informações "secundárias" como, por exemplo, o resultado dos exames realizados, não são processadas e armazenadas na memória.

O modo e contexto da apresentação da informação também podem ter influenciado o resultado. Não foram oferecidos materiais por escrito, como, por exemplo, folhetos explicativos, a respeito da deficiência auditiva e resultados dos testes para que o participante pudesse retomar as informações em um outro momento, o que pode ter dificultado a retenção das mesmas (21).

Também deve ser ressaltado que os indivíduos têm mais facilidade para reter informações concretas do que abstratas e quando os tópicos são apresentados de maneira explicitamente estruturada $(7,8)$. Ainda que as ilustrações sejam facilitadoras, o conteúdo das orientações sobre a deficiência auditiva é mais abstrato do que as orientações sobre o uso e cuidados com a prótese auditiva e molde auricular, já que estes poderiam ser visualizados e manipulados pelo participante. A avaliação dos participantes deste estudo não ocorreu imediatamente após o fornecimento das orientações. Desta forma, não é possível precisar qual o efeito que a experiência diária com o uso, cuidado e manipulação da prótese auditiva e molde auricular teve sobre a retenção das orientações dadas. No entanto, como a grande maioria dos participantes utilizou suas próteses auditivas efetivamente no período que antecedeu a avaliação é plausível afirmar que os mesmos estivessem muito mais familiarizados com este conteúdo do que sobre a sua deficiência auditiva.

Adultos evocaram significativamente mais informação do que os idosos sobre a perda auditiva, mas não sobre o uso e cuidados com a prótese. Isto pode ter ocorrido 
também em função da tarefa utilizada (evocação livre x evocação auxiliada). ReEse e HnAth-Chisolm (2005) (10) também não encontraram influência da idade na quantidade da informação retida sobre o uso e cuidados com a prótese auditiva quando tarefas de reconhecimento da informação foram utilizadas. Pesquisas em outras áreas de saúde onde foi utilizada a tarefa de evocação livre da informação também encontraram piores resultados para idosos (22,23).

Indivíduos idosos possuem pior memória para eventos ou fatos (memória episódica) podendo isto ser resultante da diminuição da capacidade cognitiva relacionadas à idade. É sugerido que a dificuldade dos idosos encontra-se mais no processo de codificação e armazenamento das informações (8).

Nãoforam observadas correlações significativas entre a escolaridade e classificação sócio-econômica com a quantidade de informação evocada. Outros estudos demonstraram aumento da retenção da informação com o aumento da escolaridade $(22,23)$. No presente estudo a influência da escolaridade pode não ter sido observada em função de a casuística ter sido relativamente homogênea, istoé, $50 \%$ dos participantes possuíam no máximo o ensino fundamental. O mesmo pode ter ocorrido com relação à classificação socioeconômica já que 63\% dos participantes encontravam-se na classe baixa superior.

Pelo fato da informação ter sido transmitida verbalmente uma das hipóteses levantadas neste estudo era a de que a severidade da perda auditiva poderia diminuir a retenção da informação pelo fato de dificultar o processo de recepção da mensagem. No entanto não foram observadas correlações entre o grau da perda auditiva e a quantidade de informação evocada pelos participantes. Tal fato pode ter ocorrido em virtude da casuística ser composta em maioria por indivíduos com perda auditiva de grau moderado ou, ainda, pelo fato de que as estratégias de comunicação utilizadas pelo profissional foram eficientes para compensar a dificuldade auditiva e, consequentemente, transmitir a informação necessária.

A retenção e compreensão das informações a respeito das características da deficiência auditiva e do uso e cuidados com a prótese auditiva e/ou molde auricular permite que os novos usuários destes dispositivos consigam extrair o máximo proveito dos mesmos bem como do processo de reabilitação. Existe um grande número de pesquisas sobre como melhorar a evocação de orientações dadas por profissionais de saúde. Foi demonstrado, por exemplo, que a retenção da informação aumenta quando os profissionais utilizam linguagem mais acessível, reconhecem a importância do aconselhamento em sua prática clínica e possuem maior auto-crítica sobre sua capacidade de transmitir informações. Também há aumento da informação retida quando as orientações fornecidas são compatíveis com as prioridades e necessidades de informação do paciente, sendo apresentadas de forma organizada com resumo e repetição dos conteúdos mais importantes. Além disto, é necessário o fornecimento de materiais educacionais (folhetos, vídeos, websites) para consulta posterior (79,21).

\section{CONCLUSÃO}

O presente estudo demonstrou que existe uma perda da informação fornecida sobre o uso e cuidados com a prótese auditiva e sobre a perda auditiva, sendo esta última maior para indivíduos idosos. Faz-se necessária à utilização de estratégias que facilitem a retenção destas orientações. Além disto, é importante a realização de outras pesquisas para investigar a influência de dados demográficos como a escolaridade no processo de retenção de informação.

\section{REFERÊNCIAS BIBLIGOGRÁFICAS}

1. Margolis RH. Page 10: What do your patients remember? The Hearing Journal. 2004, 7:10-17.

2. Lori P. Conveying information to patients. In: Sweetow, $\mathrm{R}$ (ed). Counseling for hearing aid fittings. San Diego: Singular Publishing Group; 1999; pp. 83-109.

3. Kemker BE, Holmes AE. Analysis of prefitting versus posfitting hearing aid orientation using the Glasgow hearing aid benefit profile (GHABP). JAm Acad Audiol. 2004, 15:31123.

4. Mormer E, Palmer C. A systematic program for hearing aid orientation and adjustment. Em: Sweetow, R (ed). Counseling for hearing aid fittings. San Diego: Singular Publishing Group; 1999;pp.165-199.

5. Desjardins JL, Doherty KA. Do experienced hearing aid users know how to use their hearing aids correctly? Am J Audiol. 2009, 18:69-76.

6. Watson PWB, McKinstry P. A systematic review of interventions to improve recall of medical advice in healthcare consultations. J R Soc Med. 2009, 102:235-243.

7. Ley P. Memory for medical information.Br J Soc Clin Psychol. 1979, 18:245-55.

8. Kessels RPC. Patients' memory for medical information. J Roy Soc Med. 2003, 96:219-22. 
9. Lukoschek P, Fazzari M, MarantzP. Patient and physician factors predict patients comprehension of health information. Patient Educ Couns. 2003, 50:201-10.

10. Resse JL, Hnalth-Chilson T. Recognition of hearing aid orientation content by first-time users. Am J of Audiol. 2005, 14:94-104.

11. Reese JL, Smith SL. Recall of hearing aid orientation content by first-time hearing aid users. Sem Hear. 2006, 27:337-344.

12. Oliveira JRM, Motti TFG, Neuber DRD, Prado EP, Creppe SVTZ, Andrade CF. Dificuldades encontradas pelos deficientes auditivos adultos, após a adaptação do aparelho de amplificação sonora individual (AASI). Acta AWHO. 2001, 20:201-205.

13. Freitas CD, Costa MJ. Processo de adaptação de próteses auditivas em usuários atendidos em uma instituição pública federal - parte 1: resultados e implicações com o uso da amplificação. Rev Bras Otorrinolaringol. 2007, 73:744-51.

14. Baumfield A, Dillon H. Factors affecting the use and perceived benefit of ITE and BTE hearing aids. Br J of Audiol. 2001, 35:247-58.

15. Humes L. Hearing-aid outcome measures in older adults. Proceedings of the International Conference, A Sound Foundation Through Early Amplification, Chicago, 2006. p. $265-276$.

16. World Health Organization. Prevention of blindness and deafness. Grades of hearing impairment . Disponível em: http://www.who.int/pbd/deafness/hearing_impairment_ grades/en. Acesso em 08 de novembro de 2010.
17. Graciano MIG, Lehfeld NAS, Neves Filho A. Critério de avaliação para classificação socioeconômica: elementos de atualização II. Serviço Social Realid. 1996, 5:171-201.

18. Jansen J, Butow PN, van Weert JCM, Van Dulmen S, Devine RJ, Heeren TH, Bensing JM, Tattersall M. Does age really matter? Recall of information presented to newly referred patients with cancer. J Clin Oncol. 2008, 26:54507.

19. Engel KG, Heisler M, Smith DM, Robinson CH, Forman JH, Ubel PA.Patient comprehension of emergency department care and instructions: are patients aware of when they do not understand? Ann Emerg Med. 2009, 53:454461.

20. Martin, FN, Krall I, O'Neal J. The diagnosis of acquired hearing loss: patient reactions. ASHA. 1989, 31:47-50.

21. Chan Y, Irish JC, Wood SJ, Rotstein LE, Brown DH, Gullane PJ, Lockwood GA. Patient education and informed consent in head and neck surgery. Arch Otolaryngol Head Neck Surg. 2002, 128:1269-74.

22. Hekkenberg RJ, Irish JC, Rotstein LE, Brown DH, Gullane PJ. Informed consent in head and neck surgery: how much do patients actually remember? J Otolaryngol. 1997, 26:1559.

23. Ayotte BJ, Allaire JC, Bosworth $\mathrm{H}$. The associations of patient demographic characteristics and health information ecall: the mediating role of health literacy. Neuropsychol Dev Cogn B Aging Neuropsychol Cogn. 2009, 16:419-32. 